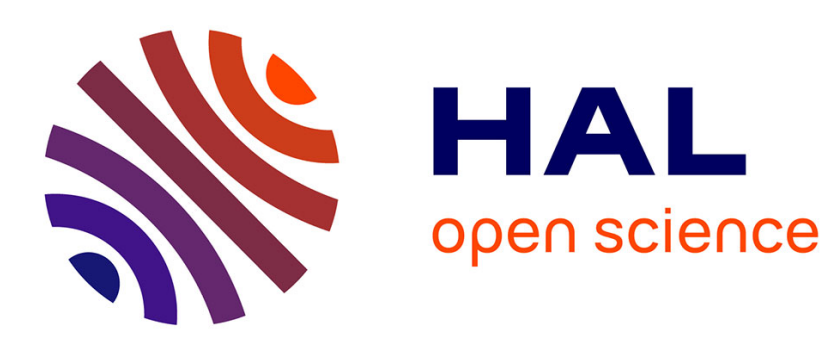

\title{
La gouvernance de médiation comme réponse aux impasses conceptuelles et pratiques de la gouvernance actionnariale
}

\author{
Xavier Hollandts, Bertrand Valiorgue
}

\section{- To cite this version:}

Xavier Hollandts, Bertrand Valiorgue. La gouvernance de médiation comme réponse aux impasses conceptuelles et pratiques de la gouvernance actionnariale. Les grands courants en management stratégique, pp.221-248, 2019. hal-03041045

\section{HAL Id: hal-03041045 \\ https://hal.science/hal-03041045}

Submitted on 4 Dec 2020

HAL is a multi-disciplinary open access archive for the deposit and dissemination of scientific research documents, whether they are published or not. The documents may come from teaching and research institutions in France or abroad, or from public or private research centers.
L'archive ouverte pluridisciplinaire HAL, est destinée au dépôt et à la diffusion de documents scientifiques de niveau recherche, publiés ou non, émanant des établissements d'enseignement et de recherche français ou étrangers, des laboratoires publics ou privés. 


\title{
La gouvernance de médiation comme réponse aux impasses conceptuelles et pratiques de la gouvernance actionnariale
}

\author{
Xavier Hollandts \\ Kedge Business School - CRCGM - Chaire Alter-Gouvernance

\section{Bertrand Valiorgue} \\ Université Clermont Auvergne - CRCGM - Chaire Alter-Gouvernance
}

La question de la gouvernance des entreprises et en particulier des bonnes pratiques de gouvernance traverse en continue l'actualité politique et médiatique. Les débats et controverses qui animent les différentes prises de position sont sous-tendues par des référentiels théoriques qui orientent la perception, le jugement et l'action des acteurs impliqués directement dans la fabrique de la stratégie des entreprises (Gomez, 2003). Depuis plusieurs décennies, c'est le tropisme de la gouvernance actionnariale stabilisé autour de la théorie de l'agence qui domine de manière hégémonique les débats (Gomez, 1996; L'Hélias, 1997; Pérez, 2010; Segrestin \& Hatchuel, 2012). Construite au carrefour de la finance et de l'économie, la théorie de l'agence (Jensen et Meckling, 1976) oriente les pratiques de gouvernance à travers la production de lois, la rédaction de codes de bonnes conduites ou encore la formation des dirigeants et administrateurs (Segrestin \& Hatchuel, 2012). C'est sans aucun doute l'une des théories issues des sciences sociales les plus performatives ${ }^{1}$ tant les implications du cadre théorisé à la fin des années 1970 conduisent à structurer et impacter une multitude de comportements collectifs et individuels (Lan \& Heracleous, 2010; MacKenzie, Muniesa, \& Siu, 2007). En ce qui concerne plus spécifiquement les sciences de gestion, l'impact de la théorie de l'agence et du modèle de gouvernance actionnariale sur la vie des entreprises est sans équivalent (Martinet \& Reynaud, 2015). Elle détermine à la fois la réflexion stratégique et sa mise en œuvre, en faisant des objectifs financiers et actionnariaux l'alpha et l'oméga de la stratégie des entreprises (Connelly, Hoskisson, Tihanyi, \& Certo, 2010; Martinet \& Reynaud, 2015; Rajan \& Zingales, 2003). Le modèle actionnarial oriente également la structure de l'entreprise en proposant de se spécialiser sur les domaines activités

\footnotetext{
${ }^{1}$ Voir le chapitre de Laure Cabantous consacré à la performativité dans ce même ouvrage.
} 
susceptibles de générer un maximum de valeur ajoutée pour les actionnaires. Elle inspire enfin la stabilisation et la mise en œuvre d'une multitude d'outils et procédures de gestion (tableaux de bords, objectifs individuels et collectifs) pour s'assurer que tout un chacun dans l'entreprise participe à l'œuvre collective de maximisation du profit pour des actionnaires réputés propriétaires de l'entreprise (Davis, 2009).

Dans ce chapitre, nous souhaitons revenir sur l'émergence et l'institutionnalisation progressive de ce référentiel théorique en matière de gouvernance des entreprises au cours du $\mathrm{XX}^{\text {ème }}$ siècle. Nous nous attardons en particulier sur les prémices de ce référentiel théorique et soulignons à la suite de nombreux auteurs sa grande fragilité (Robé, 1999, 2011b; Segrestin \& Hatchuel, 2012; Segrestin, Roger, \& Vernac, 2014). Nous mettons également en évidence les contresens à partir desquels les théoriciens de l'agence ont bâti leurs raisonnements (Blair, 1996; Blair \& Stout, 1999; Chassagnon \& Hollandts, 2014; Gindis, 2007, 2016; Robé, 1999, 2011b; Veldman \& Willmott, 2017). Dans une troisième partie, nous présentons le cadre théorique de la gouvernance de médiation qui est susceptible selon nous d'offrir une approche renouvelée de la gouvernance permettant de : définir un véritable horizon stratégique pour l'entreprise et ses parties prenantes (1) et répondre aux impasses du modèle actionnarial qui influencent les pratiques et les jugements des acteurs de la gouvernance d'entreprise (2) (Heracleous \& Lan, 2012; Rajan \& Zingales, 2003; Segrestin \& Hatchuel, 2009; Zingales, 2000). Nous concluons par une exposition rapide des grands axes de recherche susceptibles de renforcer les connaissances et assurer une plus grande performativité de la gouvernance de médiation.

\section{Fondements historiques et institutionnalisation de la gouvernance actionnariale au cours du XXème siècle}

La problématique de la gouvernance des entreprises est apparue progressivement dans l'histoire économique avec la délégation progressive de la conduite des affaires à une tierce partie. Jusqu'au $19^{\mathrm{e}}$ siècle, les entreprises étaient à la fois gérées et détenues par les mêmes personnes et la confusion de ces deux fonctions n'engendrait pas de problématiques de gouvernance telles que nous les connaissons aujourd'hui (Berle \& Means, 1932; Ripert, 1946; Segrestin, 2011). En effet, les entreprises étaient bien gouvernées mais par des personnes détenant le capital et exerçant simultanément l'autorité de gestion. 
Les spécialistes font remonter les premiers cas problématiques de gouvernance au 17/18 ème siècle (Frentrop, 2003) notamment dans le cadre des expéditions maritimes liées en partie au commerce triangulaire ${ }^{2}$ (Silverman \& Ingram, 2017).

Le cas de ces expéditions maritimes résume bien la problématique centrale de la gouvernance telle qu'elle s'est posée de manière historique et se pose toujours à l'heure actuelle. Pour effectuer ces liaisons maritimes deux cas de figures pouvaient se présenter. Premièrement, un armateur (ou un «collectif» d'armateurs) affrétait un bateau et en confiait le transport à un capitaine de vaisseau. L'armateur possédait le bateau (les actifs) et en confiait l'usage au capitaine et à son équipage (le management). Les risques pour l'armateur étaient que le capitaine ne soit pas, pour de multiples raisons (incompétence, piraterie, conditions météo, etc..) en capacité d'effectuer le trajet, de réaliser les opérations commerciales et de ramener à bon port le navire. L'armateur, en confiant la gestion de son bateau au capitaine pouvait potentiellement tout perdre, ses actifs et la valeur de la marchandise. La solution qui fut communément admise et mise en œuvre est à la fois évidente et efficace. Les armateurs décidèrent de confier une partie des bénéfices ${ }^{3}$ de la course maritime au capitaine, à condition qu'il effectue le trajet dans sa totalité et qu'il ramène le navire à son port de départ (Silverman \& Ingram, 2017). Ainsi, les armateurs s'assuraient de la convergence mutuelle des intérêts entre l'équipage et eux. Les armateurs avaient ainsi «inventé » les premières «stockoptions » de l'histoire afin de limiter les comportements opportunistes et aligner les intérêts de l'armateur et du capitaine ! Un deuxième cas, détaillé par Silverman et Ingram (2017), se rapporte au cas des capitaines, propriétaires de leurs propres navires et qui de ce fait «louaient » leurs services aux armateurs et commerçants. Dans ce cas, la détention des actifs par le capitaine a conduit à des comportements sensiblement différents. Les données exploitées par les deux auteurs montrent sur une longue période (1744-1785) que les capitaines, propriétaires de leurs propres navires, étaient plus susceptibles de renoncer à certaines expéditions trop dangereuses (guerre, piraterie) afin de préserver la sécurité du bateau. Ils se faisaient également moins capturer par des pirates ou des corsaires que les autres

\footnotetext{
${ }^{2}$ Le commerce triangulaire est avant tout une traite négrière menée au moyen d'échanges entre l'Europe, l'Afrique et les Amériques. Les bateaux partaient d'Europe, faisaient escale en Afrique afin de transporter des esclaves dans les colonies du Nouveau Monde (continent américain) et repartaient en Europe avec des produits issus des productions locales produits (sucre, café, coton, cacao, tabac).

${ }^{3}$ Une lettre adressée par un armateur à un capitaine de vaisseau en 1771 et qui détaille les incitations et conditions de rémunération contient ce passage éclairant «it's suits as much your interest as ours to bring a good and healthy cargo » (Rediker 2007 cité in Silverman \& Ingram 2017). Le système d'incitations des capitaines était basé sur une commission sur le chiffre d'affaires mais également sur un pourcentage (2\% à $6 \%$ en moyenne) de la valeur de la marchandise (voir Silverman \& Ingram, 2017, p. 861 pour plus de détails). En général, le capitaine disposait d'une rémunération totale 10 fois supérieure à celle d'un membre d'équipage.
} 
capitaines. Ainsi, le fait de risquer leurs actifs voire leur vie, incitait les capitaines à ne pas entreprendre d'expéditions risquées ou bien à modifier leur route voire à reporter leur voyage. La possession des actifs (la détention d'un capital pouvant créer de la valeur) modifiait ainsi sensiblement le comportement et les actions des capitaines qui développaient un sens plus grand des responsabilités (Silverman \& Ingram, 2017).

Les expéditions maritimes du $18^{\text {ème }}$ siècle illustrent ainsi la problématique essentielle de la gouvernance des organisations : confier la gestion d'un capital et des actifs à une tierce personne ou un collectif qui ne possède pas de droits légitimes sur ces mêmes actifs. Dans ce cas, comment s'assurer que la gestion et l'utilisation des actifs se fera dans l'intérêt des «propriétaires ». Ce qui est vrai pour les capitaines de navire, l'est aussi pour les salariés ou bien les dirigeants exécutifs. C'est bien que ce que l'on appelle la séparation entre la «propriété » et le contrôle (l'exercice de l'autorité de gestion) qui est au cœur des problématiques de gouvernance. Hormis le cas historique des transports maritime, il faudra attendre réellement l'entre-deux guerre pour que cette problématique commence à s'imposer réellement dans la vie des entreprises (Herrigel, 2007). Sous l'effet conjugué de différentes évolutions (élargissement des marchés, ouverture des capitaux, recherche de compétences et surtout passage au «factory system » et la division «scientifique » du travail), les entreprises, traditionnellement familiales, se mettent en quête de compétences, qu'elles vont chercher en dehors du cercle familial (Gomez, 2001). Les propriétaires confient alors progressivement l'orientation et la conduite des affaires à des cohortes de managers professionnels ${ }^{4}$ (Segrestin, 2011). L'ouvrage majeur de Berle \& Means, qui paraît en 1932 viendra cristalliser les problématiques essentielles de gouvernance et constituera la référence incontournable sur cette thématique. Le second tournant se situe dans les années 1970 avec l'émergence de la primauté actionnariale et la théorie de l'agence. C'est ce référentiel théorique qui oriente et domine depuis la fin des années 1970 les débats et pratiques en matière de gouvernance des entreprises.

\footnotetext{
${ }^{4}$ Les historiens font remonter à l'entre-deux guerres, la prise de pouvoir progressive de managers professionnels, embauchés par des actionnaires, souvent familiaux. Comme l'affirme le président Roosevelt en 1932 «the day of the manager has come » (discours du 23/9/1932 in Segrestin, 2011).
} 


\subsection{Berle et Means 1932 : la séparation propriété/contrôle et le débat gouvernance actionnariale vs partenariale}

En 1932, parait l'ouvrage rédigé par Adolph Berle et Gardiner Means qui constitue la référence incontournable sur la gouvernance des entreprises (Berle \& Means, 1932) ${ }^{5}$. La communauté académique fait souvent remonter à cet ouvrage la mise en évidence des problématiques de gouvernance telles que nous les connaissons à l'heure actuelle (Segrestin, $2011)^{6}$. Il doit cependant être resitué dans le cadre d'un débat juridique intense, qui s'est soldé in fine par l'émergence de la suprématie actionnariale (Blair, 1996). Durant l'entre-deux guerres, se pose à la fois la question de la gestion des entreprises, de la séparation progressive entre propriété et contrôle mais également une question plus essentielle : dans quel intérêt une entreprise doit-elle être gérée?

\subsection{Au profit de qui une entreprise doit-elle être gouvernée?}

Sur cette question, le droit des sociétés américain, essentiellement jurisprudentiel, s'est construit au travers de décisions de justices majeures comme le célèbre arrêt Dodge vs Ford de la Cour Suprême de l'Etat de Michigan (170 N.W. 668 Michigan 1919 - (Pour une discussion critique voir Stout, 2008). Cet arrêt, qui est l'un des plus commentés et l'un des plus étudiés par les juristes américains, constitue le point de départ jurisprudentiel de la reconnaissance de la suprématie actionnariale dans le droit américain (pour une mise en contexte historique, voir Fish, 2006 ; Smith et Tennent, 2017). Cette affaire opposa à l'époque deux frères, actionnaires minoritaires de l'entreprise Ford, qui soulignaient que l'entreprise aurait dû verser un dividende plus important à ses actionnaires, de l'ordre de 10 millions de dollars. La position d'Henry Ford était plutôt de prétendre qu'il était de la responsabilité des dirigeants et des administrateurs de décider de l'affectation et de la répartition du résultat et que son ambition à l'époque était d'employer plus de salariés, de partager le plus possible les profits et de les réinvestir dans l'entreprise afin de la faire prospérer. Cette vision n'était pas partagée par les deux frères, Horace et John Dodge qui considéraient que les dividendes

\footnotetext{
${ }^{5}$ L'ouvrage a été rédigé en plusieurs temps. L'introduction et les derniers chapitres n'ont ainsi été rédigés que bien plus tard après le cœur de l'ouvrage (Segrestin 2011). Le livre comporte au final 4 sections. La première est consacrée à une analyse historique, qui met en évidence la séparation propriété/contrôle. La seconde examine le droit des différentes parties dans ce contexte. La troisième porte sur le fonctionnement et la régulation des marchés financiers. Enfin, la dernière section s'attache à mettre en avant la nécessité de refonder les principaux concepts du droit et de l'économie.

${ }^{6}$ Au-delà du champ de la gouvernance, l'ouvrage a eu une influence considérable aux Etats-Unis et dans le monde. Entre 1996 et 2016, l'ouvrage est cité au moins 8 fois/an dans les pages du New York Times ainsi que dans de nombreux arrêts et expertises judiciaires (Smith \& Tennent 2017).
} 
proposés ne représentaient qu'une fraction très minime des bénéfices dégagés par Ford. Ce conflit qui, à l'origine, portait plus sur le pouvoir exorbitant de l'actionnaire majoritaire contrôlant Ford par rapport aux actionnaires minoritaires, offrit l'opportunité au juge de jeter les bases juridiques de la suprématie actionnariale. L'arrêt qui condamne Ford et qui se range aux arguments des frères Dodge comporte un passage éclairant, très souvent repris dans les manuels de droit et ouvrages juridiques : «There should be no confusion. A business corporation is organized and carried on primarily for the profit of the stockholders. The powers of the directors are to be employed for that end. The discretion of the directors is to be exercised in the choice of means to attain that end, and does not extend to other purposes » (Dodge vs Ford Motor Co., 170 N.W. 668 Michigan 1919) ${ }^{7}$. C'est cet arrêt Dodge vs Ford de 1919 qui ouvre les débats contemporains sur la gouvernance des entreprises car il prend acte de la dilution de l'actionnariat des grandes entreprises et de leur perte d'influence progressive par rapport aux managers professionnels qui progressivement prennent en charge la construction et la mise en œuvre de la stratégie. Cet arrêt servira de point de départ au questionnement de Berle et Means et alimentera toute une série de débats durant l'entre-deuxguerres sur la finalité du profit généré par l'entreprise.

\subsubsection{Les débats de l'entre-deux-guerres}

L'arrêt Dodge vs Ford revêt une grande importance dans la construction jurisprudentielle de la suprématie actionnariale, même si comme le rappelle Stout (2002), le débat continuera à faire rage pendant de nombreuses années et la plus célèbre controverse oppose les Professeurs Berle et $\operatorname{Dodd}^{8}$ en 1931/1932 dans la Harvard Law Review. Le Pr Berle défend une approche plutôt actionnariale. Il précise par exemple: «all powers granted to a corporation or to the management of a corporation [are] at all times exercisable only for the ratable benefit of the shareholders" (Berle 1931). La position de Berle semble avoir évolué dans le temps et l'analyse de sa correspondance montre que par certains côtés, Berle a pu envisager une perspective élargie de la gouvernance (Smith \& Tennent 2017 - voir note de bas de page $\mathrm{n}^{\circ}$ ). Le Pr Dodd défend quant à lui une approche clairement partenariale de la gouvernance (stakeholderism) et souligne que l'entreprise doit être gérée par les dirigeants dans l'intérêt

\footnotetext{
${ }^{7}$ https://pages.law.illinois.edu/aviram/Dodge.pdf

${ }^{8}$ Ce débat, à distance, opposera en fait pendant plusieurs années les deux juristes. Les spécialistes estiment que quatre articles essentiels peuvent être versés au débat Berle/Dodd (deux écrits par Berle en 1931/1932 et deux par Dodd en 1932 et 1935). Les deux articles majeurs sont : Berle A., 1931. Corporate powers as powers in trust. Harvard Law Review 1931, 44:1049-1074 et Dodd E. (1932). For whom are corporate managers trustees. Harvard Law Review 1932, 45:1145-1163.
} 
d'une communauté plus large composée des salariés, des clients et de la collectivité en général. Pour Dodd, les entreprises ne doivent pas être gérées dans le seul intérêt des actionnaires: " the business corporation has a social service as well as a profit-making function » (Dodd, 1932 :1148). Berle et Means (1932:5) n'écartent pas complètement cette vision élargie mais soulignent que la problématique essentielle de la gouvernance met aux prises les actionnaires et les dirigeants. Or, dès l'entre-deux guerres les actionnaires se montrent de plus en plus passifs (ils n'ont plus le contrôle des opérations) et dépendent de facto des actions et de la stratégie des managers. Ils cherchent surtout à souligner que certaines dérives «managériales» peuvent être problématiques. Cette opposition théorique sur la nature juridique et politique de l'entreprise (la société) reste encore vivace, y compris dans les Law schools américaines où partisans et opposants de l'approche actionnariale continuent parfois à s'affronter (Stout 2008). A l'heure actuelle, deux approches du droit des sociétés co-existent.

D'un côté les tenants de l'approche contractarian qui considèrent que les actionnaires possèdent la firme/la société et que les dirigeants sont les intendants fidèles ( «mere stewards »), garants du respect des intérêts des actionnaires (Bainbridge 2008:8). Pour les partisans de cette approche, le rôle du droit des sociétés est de minimiser les problèmes d'agence résultant des conflits d'intérêts potentiels entre actionnaires et dirigeants (Fish 2006). Les individus mus par leur propre intérêt, cherchent avant tout à tirer parti des failles des différents contrats ; il revient alors au droit des sociétés de permettre le traitement contractuel ou judiciaire de ces problèmes d'agence (Fish 2006). Ce cadre de référence constitue, y compris de l'aveu de certains juges (Bainbridge 2008 :30), le modèle dominant, certes imparfait, en droit américain des sociétés.

A l'autre extrémité figurent les partisans d'une approche partenariale (communitarian ou stakholderism), dont Dodd fut le lointain précurseur, qui considèrent que l'entreprise est un ensemble complexe de relations explicites (contractuelles) et implicites avec plusieurs parties prenantes et que l'entreprise doit être gérée dans l'intérêt de l'ensemble des parties prenantes dont l'entreprise elle-même (Lan et Heracleous 2010). Ainsi, dès 1931/32, le débat opposant Dodd à Berle et Means préfigure déjà les débats ultérieurs opposant les partisans d'une approche actionnariale à ceux défendant une approche plus inclusive/partenariale (Charreaux \& Desbrières, 1998). 
Une lecture rapide de l'ouvrage de Berle et Means pourrait laisser penser que la thèse des auteurs se réduit à la question de la séparation de la propriété et du contrôle mais résumer l'ouvrage à cette unique thèse est à la fois faux et caricatural (Segrestin, 2011). Tout en rappelant la place prépondérante des actionnaires, les deux auteurs invitent plutôt à considérer les conséquences du développement du management et des managers dans les entreprises de l'époque (Magnan de Bornier, 1987 ; Segrestin, 2011) ${ }^{9}$. En effet, leur interrogation essentielle est plutôt d'imaginer les conséquences potentielles et les adaptations nécessaires (notamment juridiques) de la séparation progressive entre propriété et contrôle. Ainsi, leur ouvrage témoigne à la fois de la dispersion actionnariale, qu'ils observent notamment d'un point de vue statistique ${ }^{10}$ mais également du début d'une nouvelle ère, que l'on qualifiera par la suite de «révolution managériale » (voir les travaux de Chandler à ce sujet notamment). Tout au long de leur ouvrage, Berle et Means développement une thèse « ambivalente ». Ils font certes le constat de cette séparation entre propriété du capital et direction effective de l'entreprise. Mais selon eux, ce nouveau contexte n'est pas nécessairement négatif. En effet, les entreprises managériales, plus adaptées aux transformations économiques que les auteurs observent, sont susceptibles d'être potentiellement des organisations plus performantes. L'origine de la création de valeur change et s'appuie aussi bien sur des méthodes modernes de production et de management et nécessitent par conséquent d'être orchestrées par des dirigeants «professionnels ». Après la seconde guerre mondiale, les problématiques de gouvernance passeront au second plan car les entreprises managériales affichent à cette époque des résultats économiques très satisfaisants. Les équilibres entre les actionnaires et les dirigeants professionnels ne sont pas contestés.

\subsection{La primauté actionnariale réaffirmée via la théorie de l'agence}

L'émergence progressive de la théorie de l'agence dans les années 1970, qui marque le retour de l'actionnaire, semble liée à la chute brutale du niveau de profits des entreprises américaines dans les années 1970 (Lazonick \& O'sullivan, 2000). C'est cette baisse de productivité des entreprises américaines en particulier à l'égard des entreprises japonaises qui ré-ouvre le débat sur la gouvernance et les pratiques à mettre en œuvre pour inciter les dirigeants à mettre

\footnotetext{
${ }^{9}$ Leur doctrine à ce sujet est complexe à saisir, principalement car leur ouvrage semble avoir été constitué par «ajouts successifs ». Pour Smith \& Tennent (2017), qui ont travaillé sur la correspondance de Berle \& Means, il semble qu'ils aient également partagé une approche plus plurielle de la gouvernance, qui n'est pas nécessairement retranscrite dans la version finale de leur ouvrage.

${ }^{10}$ Leur étude statistique, portant sur 200 grandes entreprises, révèle que dans 44 à $58 \%$ de ces entreprises, aucun individu n'est en position d'avoir une part suffisante pour contrôler la société (Berle et Means, 1932, p. 109 in Segrestin, 2011, p. 97).
} 
en place des stratégies plus compétitives. Cette refonte de la gouvernance et des équilibres actionnaires - dirigeants va se faire autour de la théorique de l'agence qui va constituer le cadre théorique dominant permettant à la fois d'incarner les principales problématiques de gouvernance tout en justifiant la suprématie supposée des actionnaires. En effet, la problématique mise au jour par Berle et Means (la séparation propriété /contrôle) va dès lors être généralement analysée comme une relation d'agence entre actionnaires et dirigeants. Reprenant à leur compte certains passages de l'ouvrage de Berle et Means, les théoriciens de l'agence n'en retiendront qu'une lecture partielle et très partiale (Segrestin, 2011 : p. 99). L'idée que l'entreprise doit alors prioritairement être gérée dans l'intérêt des actionnaires s'impose alors peu à peu, tant dans le champ académique (Friedman, Fama, Jensen, Meckling) que dans le monde des affaires (Smith et Tennent, 2017). Les équilibres trouvés pendant la période fordiste volent en éclat au profit d'une domination progressive puis hégémonique des actionnaires réputés propriétaires de l'entreprise.

Pour les théoriciens de l'agence, les actionnaires délèguent leur autorité/pouvoir légitime de direction (en tant que «propriétaires légitimes ») aux dirigeants, qui dirigent concrètement l'entreprise. Cependant les dirigeants peuvent avoir des objectifs potentiellement différents de ceux des actionnaires (des intérêts divergents), à l'instar de nos armateurs et capitaines de navire. Ces conflits potentiels peuvent porter sur la stratégie de l'entreprise, ses objectifs, la distribution des profits mais également sur les relations avec les parties prenantes. Ces divergences potentielles, sont alors dénommées «conflits d'agence » et constituent la problématique standard de la gouvernance d'entreprise (Moore et Reberioux, 2010). La qualité de la surveillance (exercée par les actionnaires) sur ceux qui contrôlent l'entreprise (les dirigeants) devient essentielle à la bonne gouvernance de l'entreprise. Dans ce système, les actionnaires sont placés en «surplomb» des autres parties prenantes, au motif qu'ils apparaissent aux yeux de beaucoup comme les «propriétaires » de l'entreprise (Chassagnon \& Hollandts, 2014). Dans cette perspective, la gouvernance d'entreprise vise à encadrer et inciter les dirigeants à avoir des comportements adéquats, c'est-à-dire, créant (ou ne détruisant pas) de valeur pour l'actionnaire (Charreaux, 1997; Fama \& Jensen, 1983). La théorie de l'agence est marquée par la thèse de la dépossession (où comment le «pouvoir » peut-il être reconquis ?) et elle tente de sanctuariser la place des actionnaires dans la gouvernance des entreprises (Hansmann \& Kraakman, 2001)

Cette théorie de la gouvernance qui se construit essentiellement autour des travaux de Fama, Jensen et Meckling puise ses racines dans la théorie des droits de propriété qui offre le 
soubassement théorique nécessaire comme le reconnaissent Jensen et Meckling (1976). L'entreprise n'est alors présentée que comme «fiction légale $»^{11}$. Dit autrement, l'entreprise n'as pas réellement d'existence ; c'est une abstraction juridique qui permet le déroulement des activités économiques principales. Seuls existent les contrats formels et informels, passés entre les possesseurs des facteurs de production, les fournisseurs et les consommateurs (Fama \& Jensen, 1983; Furubotn \& Pejovich, 1972). La firme sert alors d'habillage juridique à un processus complexe par lequel les objectifs conflictuels des individus sont conduits à un équilibre à l'intérieur d'un cadre constitué de relations contractuelles (Jensen \& Meckling, 1976). La théorie de l'agence, qui se base explicitement sur la théorie des droits de propriété, va ainsi, par approximations successives, permettre aux actionnaires de se présenter comme les propriétaires «légitimes » de l'entreprise et les placer dans une position prépondérante, quelle que soit le type de gouvernance considérée.

\subsection{Qu'est-ce qu'une entreprise bien gouvernée ?}

Dans cette approche, la bonne gouvernance de l'entreprise dépend directement de la qualité et du dosage de la surveillance des dirigeants mais également des incitations censées les orienter. Si le système d'incitations/sanctions est efficace, l'entreprise est alors susceptible de créer plus de valeur actionnariale (Fama \& Jensen, 1983). Dans le cadre de ce schéma de gouvernance, les actionnaires apportent le capital nécessaire au bon fonctionnement de l'entreprise et supportent le risque associé. Ils détiennent de ce fait le droit de nommer, surveiller, récompenser et sanctionner les dirigeants qui utilisent les droits associés à la détention du capital. Mais ces dirigeants peuvent être enclin à promouvoir leurs propres intérêts (égoïstes) cherchent avant tout à tirer parti des failles des relations contractuelles les liant aux actionnaires. Il revient alors au droit des sociétés de permettre le traitement contractuel ou judiciaire de ces problèmes d'agence et limiter au maximum les risques de comportement opportunistes des dirigeants mandatés par les actionnaires pour servir leurs intérêts (Fish, 2006). Ce cadre de référence constitue, y compris de l'aveu de certains juges (Bainbridge, 2008 : p. 30), le modèle dominant, certes imparfait, en droit américain des sociétés. Il repose in fine, sur un diptyque solide caractérisé par (1) la norme de maximisation de la valeur actionnariale et (2) le principe de contrôle en dernier ressort de l'actionnaire via le conseil d'administration (Bainbridge, 2008). Or, ce modèle, même s'il est parfois attaqué

\footnotetext{
${ }^{11}$ «It is important to recognize that most organizations are simply legal fictions (By legal fiction we mean the artificial construct under the law which allows certain organizations to be treated as individuals) which serve as a nexus for a set of contracting relationships among individuals » (Jensen et Meckling 1976, p.314).
} 
plus ou moins frontalement sur ses propres fondements a comme avantage de présenter une série d'arguments rendant légitimes la prédominance des actionnaires dans le gouvernement de l'entreprise.

Du point de vue économique et juridique, les arguments justifiant le rôle prépondérant des actionnaires se situent à trois niveaux imbriqués : les actionnaires sont les «propriétaires » légitimes de la firme (1); ils supportent un risque important (2); ils sont les créanciers résiduels (3) (voir Gomez (1996) pour une présentation des arguments économiques et Stout (2002) pour une présentation des arguments juridiques). Les actionnaires, en fournissant le capital nécessaire au démarrage de l'activité offrent ainsi le point de départ indispensable à l'établissement des relations contractuelles autour desquelles s'organise l'activité économique. Les actionnaires possèdent des droits de propriété sur le capital qui, par extension, se confond avec la firme, puisque qu'elle ne constitue pas une entité autonome (Bainbridge, 2008 : p. 32). C'est justement sur ce point que les critiques les plus fournies viseront à montrer que la personnalité morale de la firme la rend impropre à être un objet de propriété (Gindis, 2007; Robé, 1999).

Par ailleurs, les actionnaires en investissant dans la firme semblent supporter un risque important lié à leur investissement. En effet, détenir des actions ou des parts sociales, constitue une sorte de pari puisqu'ils n'ont aucune garantie sur la pérennité et la rentabilité de leur investissement. Les actionnaires sont alors supposés supporter un risque important et théoriquement le plus important par rapport aux autres parties prenantes (Easterbrook et Fishel 1991). C'est justement ce mélange de droit formel de contrôle (puisque détenteurs de « droits de propriété ») et de droit au profit résiduel qui leur confère, dans la théorie de l'agence, le statut de «propriétaire » de la firme (Hansmann 1996 :11). Dès lors, il est dans l'intérêt des actionnaires, dans une pure logique économiciste, de vouloir optimiser la gestion de l'entreprise, ce qui maximise l'efficacité économique globale (Alchian, 1961). Ainsi, émerge la notion de maximisation de la valeur actionnariale. Cet indicateur, devient par défaut (en raison de l'incomplétude contractuelle) l'indicateur principal de bonne gestion de l'entreprise, voire la boussole des actionnaires et des dirigeants (Hillman \& Keim, 2001; Lazonick \& O'sullivan, 2000). Il est dans l'intérêt «privé » des actionnaires de maximiser cette valeur mais également dans l'intérêt collectif puisque les entreprises maximisant la valeur actionnariale sont plus à même de verser des salaires plus élevés, de payer plus d'impôts et de 
taxes et donc au niveau global, par effet agrégatif, d'améliorer le bien-être social ${ }^{12}$ (Stout, 2007).

Dès lors, il paraît logique que le conseil d'administration apparaisse comme l'organe de de contrôle et d'incitation des dirigeants (Daily, Dalton, \& Cannella, 2003), afin qu'ils agissent au mieux dans les intérêts des actionnaires. L'efficacité de cette structure de gouvernance sera essentiellement garantie par l'utilisation de trois éléments essentiels : (1) l'indépendance des administrateurs vis-à-vis des dirigeants ; (2) l'alignement des intérêts des dirigeants sur ceux des actionnaires par le biais par exemple des mécanismes de stock-options ; (3) le recours au marché des prises de contrôle afin de sanctionner les comportements déviants des dirigeants (ou des conseils d'administration).

\section{Une nouvelle approche de la gouvernance : la gouvernance de médiation}

La domination hégémonique de la théorie de l'agence à partir de la fin des années 1970 est le résultat d'un long processus intellectuel et jurisprudentiel dont les prémices se retrouvent dans l'arrête Dodge vs Ford de 1919 et l'ouvrage de Berle \& Means publié en 1932. C'est seulement à partir du milieu des années 1990 que l'on voit apparaître une critique solide et argumentée qui vise à démontrer la fragilité des arguments et les contresens sur lesquelles les théoriciens de l'agence ont construit leurs raisonnements. Cette remise en cause des fondements de la théorie de l'agence se cristallise autour des travaux de juristes et économistes américains et va progressivement donner naissance à la gouvernance de médiation. Ce référentiel alternatif à la théorie de l'agence trouve ces origines au carrefour de l'économie et du droit. Il s'appuie en particulier sur la théorie des contrats incomplets (Aghion \& Holden, 2011) et la nouvelle théorie des droits de propriété (Barzel, 1997; Foss \& Foss, 2015) qui apportent les ressources conceptuelles nécessaires pour pointer du doigt les apories de la théorie de l'agence et offrir une voie de dépassement originale et conceptuellement structurée. L'article majeur exposant cette nouvelle approche, A Team Production Theory of Corporate Law, est publié par M. Blair et L. Stout en 1999 dans la Virginia Law Review (Blair \& Stout, 1999). Dans les années 2000, les travaux et publications de Rajan et Zingales ont contribué à donner une consistance renforcée et une importante visibilité à la théorie de la gouvernance de médiation (Rajan \& Zingales, 2003; Rajan \& Zingales, 1998, 2000, 2001; Zingales, 2000). Le point de vue adopté en matière de

\footnotetext{
${ }^{12}$ Cette ligne est également défendue par Friedman lorsqu'il prétend dans une célèbre tribune que l'entreprise n'a pas à supporter le coût des externalités et que son seul objectif est de maximiser le profit (M. Friedman, The Social Responsibility of Business is to Increase its Profits, New York Times Magazine, 13/9/1970).
} 
gouvernance remet frontalement en cause les préceptes de la théorie de l'agence car il est démontré que l'entreprise n'est pas la propriété des actionnaires, que sa nature n'est pas contractuelle et que le conseil d'administration ne doit pas représenter les seuls intérêts des actionnaires. La gouvernance de médiation s'oppose ainsi point par point à la théorie de l'agence et offre des perspectives nouvelles pour penser et pratiquer la gouvernance d'entreprise. Elle conduit également à profondément revoir le contenu de la stratégie et la manière dont celle-ci est opérationnalisée par les dirigeants.

\subsection{L'entreprise n'est pas la propriété de ses actionnaires}

La question de la propriété de l'entreprise est une question centrale dont l'issue semble parfaitement tranchée dans le droit et l'opinion publique. Une entreprise appartient tout simplement à ses actionnaires car le droit leur reconnaît cette prérogative. C'est eux qui apportent les capitaux nécessaires et c'est également eux qui supportent les risques liés à l'activité (Chassagnon \& Hollandts, 2014). L'appartenance de l'entreprise a ses actionnaires peut ainsi être qualifié à la suite d'Ellerman (1996) de 'mythe fondateur' tant cette vision est universellement partagée aussi bien par les thuriféraires du capitalisme que par ses détracteurs (Ellerman, 1990). Cette vision de la propriété de l'entreprise par les actionnaires qui se situe au cœur de l'ouvrage de Berle et Means et aux fondements de la théorie de l'agence est frontalement critiquée par les auteurs attachés à la théorie de la gouvernance de médiation. Les auteurs dénoncent à partir de la lecture du droit une lecture superficielle et des conclusions partisanes sur cette question fondamentale en matière de gouvernance.

La gouvernance de médiation déboulonne le mythe fondateur de la propriété et primauté actionnariales en rappelant que l'on ne peut en aucun cas affirmer que les actionnaires sont les propriétaires de l'entreprise pour la simple et bonne raison que l'entreprise n'existe pas en droit (le droit ne connait que les sociétés) et par conséquent, elle ne peut en aucun cas être un objet de propriété (Blair, 1996; Chassagnon \& Hollandts, 2014; Robé, 1999). De même que personne ne possède un État ou une association, personne ne possède une entreprise. La gouvernance de médiation rappelle qu'il y a un immense décalage entre les représentations des acteurs qui perçoivent l'entreprise comme une entité appartenant à ses actionnaires et les juristes qui ne reconnaissent pas l'entreprise en droit (Robé, 1999, 2011b; Segrestin \& Hatchuel, 2009, 2012). Cette erreur d'interprétation provient d'une confusion entre les termes de société et entreprise qui sont généralement pris pour équivalent. Si le droit ne reconnaît pas l'entreprise, il reconnaît en revanche la société qui existe en droit positif. La société doit être 
entendue comme la personne ou le véhicule juridique titulaire de droits et propriétaires d'actifs qui permet à l'entreprise de fonctionner et passer des contrats (Robé, 1999). Le droit reconnaît que les actionnaires sont propriétaires de titres de propriété sur la société (des actions) qui leur donnent le droit de percevoir des dividendes, de participer à des assemblées générales d'actionnaires et d'élire des représentants. Les actionnaires ne sont propriétaires que des actions émises par la société commerciale qui sert de support juridique à l'activité de l'entreprise. Ils ne sont propriétaires ni de la société, ni de l'entreprise (Robé, 2011b). Les auteurs du courant montrent que le raisonnement axé autour du droit de propriété et de la primauté des actionnaires est basé sur une erreur d'interprétation fondamentale qui consiste à affirmer que l'entreprise est un objet de droit de propriété. Le droit ne dit pas cela. En droit, l'entreprise n'existe pas et il n'est donc pas possible d'en être propriétaire. La gouvernance de médiation rappelle ainsi qu'être propriétaires d'actions d'une société commerciale ne revient pas à être propriétaires de l'entreprise et ne donne pas le droit d'imposer ses intérêts. Les auteurs de la gouvernance de médiation mettent en avant ce détournement et concluent à partir de la simple lecture du droit que l'entreprise n'appartient pas aux actionnaires et que les représentants qu'ils élisent ne doivent pas privilégier leurs intérêts (Blair, 1996; Blair \& Stout, 1999, 2001).

\subsection{L'entreprise n'est pas un nœud de contrats}

Après avoir démontré que l'entreprise n'est pas un objet de propriété, les auteurs s'attaquent à un deuxième pilier de la théorie de l'agence qui consiste à définir l'entreprise comme un nœud de contrats. En confondant, la société et l'entreprise, il est assez logique que la théorie de l'agence conclut que l'entreprise est une fiction légale qui sert de véhicule juridique pour signer des contrats et organiser des transactions avec différentes parties prenantes nécessaires au bon déroulement du processus économique (salariés, fournisseurs, sous-traitants, clients, etc.). Cette vision de l'entreprise comme simple nœud de contrats correspond pour les auteurs affiliés à la gouvernance de médiation à une vision trompeuse, partielle et inexacte de la nature de l'entreprise (Rajan \& Zingales, 2000, 2001; Zingales, 2000). Si effectivement de nombreux contrats sont signés par la société pour assurer l'activité de l'entreprise, cette dernière ne peut pas être vue comme un simple support de contractualisation.

Pour les auteurs attachés à la gouvernance de médiation, l'entreprise est avant tout une collection de ressources partagées, de talents et d'idées qui combinés entre eux vont générer une valeur économique via la stabilisation d'une quasi-rente organisationnelle (Rajan \& 
Zingales, 1998, p. 405). L'entreprise n'est alors pas un nœud de contrats mais un nœud d'investissements spécifiques au sein duquel différents acteurs vont investir et spécialiser des ressources/compétences/identités en vue de générer une valeur économique. Comme le précise Blair et Stout 2001, cette conception de l'entreprise reconnaît que la fonction de production de cette dernière requiert l'intégration de différentes parties prenantes : actionnaires, créanciers, prêteurs, salariés, managers et même les autorités locales peuvent être en mesure de contribuer au succès de l'entreprise (Blair \& Stout, 2001). Dès lors, la théorie de l'agence qui fait de l'entreprise une fiction légale support des relations contractuelles ne peut être satisfaisante pour reconnaître l'apport de ces différentes parties prenantes. Pour la gouvernance de médiation, l'entreprise est un nœud d'investissements spécifiques notamment en capital humain. Cette approche n'est d'ailleurs pas très éloignée de celle d'Aoki, qui définit l'entreprise comme «une combinaison stabilisée de ressources spécifiques » (Aoki, 2000). L’entreprise est ainsi conçue dans le cadre de la gouvernance de médiation comme une combinaison indissociable de capitaux financiers et humains apportés par un certain nombre de parties prenantes. La création de valeur n'est plus, comme dans la théorie de l'agence, le fruit d'une rencontre négociée et calibrée entre des apporteurs d'actifs et de compétences dont les interactions sont définies et régulées une bonne fois pour toutes par des contrats. La création de valeur est le fruit d'interactions multiples, complexes et imprévisibles entre différents catégories d'acteurs qui apportent et spécialisent des actifs, des compétences, des réseaux, des brevets, des informations... qui combinés entre eux permettent à l'entreprise de dégager une valeur économique. Il est claire pour les auteurs que la création de valeur est le résultat d'une activité productive complexe incluant plusieurs parties prenantes et dont les résultats ne sont ni séparables ni individuellement attribuables (Heracleous \& Lan, 2012; Lan \& Heracleous, 2010). Les actionnaires sont un des acteurs et ne peuvent pas logiquement être considérés comme les seuls créanciers résiduels (Blair \& Stout, 2001).

\subsection{Les administrateurs et le conseil d'administration ne représentent pas les actionnaires}

Compte tenu des évolutions substantielles sur la propriété puis la nature de l'entreprise, la théorie de la gouvernance de médiation n'est évidemment pas sans conséquences sur le rôle et la composition du conseil d'administration. Cette vision renouvelée du rôle du conseil d'administration se structure autour de deux enjeux clés : la création et le partage de la valeur économique. Contrairement à la théorie de l'agence qui se structure autour d'un règlement par 
les contrats des relations de collaboration entre les apporteurs de ressources et capitaux, la gouvernance de médiation fait ressortir et assume un paradoxe : il y a des relations de coopération pour la production de la richesse et des relations de concurrence pour sa répartition. Si ce paradoxe n'est en soit pas une découverte, la gouvernance de médiation a le mérite de le placer au cœur des enjeux de gouvernance. Sa résolution aboutit à une conceptualisation très différente du rôle du conseil d'administration par rapport à la théorie de l'agence.

Pour trouver une solution (toujours provisoire) au paradoxe de la création collective/répartition individuelle de la valeur, il est indispensable qu'une autorité de gestion émerge au sein de l'entreprise afin de garantir une combinaison efficace et spécialisée d'actifs, une implication pleine et entière des acteurs et une juste valorisation des efforts individuels (Blair \& Stout, 2001; Rajan \& Zingales, 2001). L'horizon d'une bonne gouvernance est ainsi de reconnaître, développer et valoriser les investissements bénéfiques à l'entreprise, qui, au-delà du simple investissement en capital, participent à la création de valeur et la stabilisation d'une rente organisationnelle. Ces interactions et la nécessaire dynamique de coopération ne vont bien évidemment pas de soi et la théorie de la gouvernance de médiation est bien consciente des écueils de l'action collective en matière de free riding, asymétrie d'information et sous-investissement en ressources et compétences spécifiques. Pour faire face à ces enjeux, les auteurs postulent la nécessité d'une instance de gouvernance qui va analyser, développer et réguler l'action collective entre les apporteurs de ressources et compétences spécifiques. Cette instance qui porte le nom de third part ownership chez Rajan et Zingales ou mediating hierarchy chez Blair et Stout va permettre de faire émerger et stabiliser sur la durée une coopération efficace entre les acteurs (Blair \& Stout, 2001; Rajan \& Zingales, 2001). Elle va également éviter les abus en matière de sur-captation de la valeur et sous-implication dans le processus de création. La tâche essentielle de cette instance est de sélectionner parmi les différentes catégories d'apporteurs de ressources (actionnaires, salariés, fournisseurs, etc.), celle qui produit le plus grand résultat économique. Les auteurs soulignent qu'il est incontournable de garder le contrôle des actifs et des ressources en dehors des mains des détenteurs du capital humain et du capital financier et plus généralement des investisseurs spécifiques, afin qu'ils ne s'en servent pas pour accroître leur pouvoir et capter une part trop importante de la valeur économique. Dans l'optique de Rajan et de Zingales ainsi que Blair et Stout, cette instance de gouvernance pourrait être une entité légale séparée de l'entreprise qui détiendrait les droits de propriété (ainsi que les droits de contrôle) sur les investissements 
physiques et les ressources critiques. De part ces prérogatives, cette instance de gouvernance se comporte en arbitre qui évite que les employés, les actionnaires et les parties prenantes spécifiques usent de leur pouvoir l'un contre l'autre au détriment d'une création de valeur collective.

Pour les auteurs, cette proposition ne représente pas une révolution mais au contraire une réaffirmation du rôle du conseil d'administration. Ce dernier n'a pas pour vocation de défendre les intérêts des actionnaires mais de protéger les investissements spécifiques de l'entreprise et de défendre l'intérêt social (Blair \& Stout, 1999, p. 253). De plus, les deux auteurs (Blair \& Stout, 1999, p. 253) précisent que les membres de l'entreprise se soumettent à cette autorité de gestion pour leur propre bénéfice car il s'agit de protéger leurs investissements spécifiques et encourager les membres à continuer de développer et exploiter les investissements. Le conseil d'administration doit agir dans le seul intérêt de l'entreprise afin d'éviter les décisions partiales bénéficiant avant tout aux seuls actionnaires ou aux seuls salariés dont les capitaux humains sont spécifiques.

De manière concrète, il est clair pour les auteurs attachés au courant de la gouvernance de médiation que la seule instance à même d'exercer cette fonction est le conseil d'administration. L'exercice d'une fonction neutre de décision est rendu possible par le fait que les administrateurs ne sont pas juridiquement les agents des actionnaires mais bien des individus devant exercer leurs fonctions de manière neutre et au seul regard de l'intérêt collectif et de la pérennité de la société (Blair \& Stout, 2001). Les administrateurs agissent au nom et pour le compte de la société et non pour une de ses parties prenantes, aussi « légitime » soit-elle. Sur ce point, Lan et Heracleous (2010) soulignent que nombre de décisions ont refusé de reconnaître aux actionnaires la capacité et le droit d'annuler ou de rejeter une décision prise par le conseil d'administration (Lan \& Heracleous, 2010, p. 303). Le conseil d'administration permet la représentation et l'expression d'un intérêt collectif incarnée en quelque sorte par la société (Robé, 2011b). Dans cette configuration, le conseil d'administration agit comme une instance collégiale, comme un « juge de cour d'appel » (Frey \& Osterloh, 2005, p. 104) qui résout les conflits potentiels entre actionnaires, salariés ou autres apporteurs de ressources spécifiques.

Il ressort de cette analyse que le conseil d'administration doit être majoritairement composé d'administrateurs externes ou indépendants (Frey \& Osterloh, 2005) et que le président du conseil soit lui-même un personnage neutre et non-partisan (Osterloh \& Frey, 2006) afin de 
garantir le fonctionnement correct. Le président du conseil aura pour mission de faire scrupuleusement respecter les règles de procédures et devra dans la mesure du possible chercher à faire émerger un consensus. Cette personne, afin de garantir sa neutralité (à l'instar d'un juge vis-à-vis d'un jury), ne doit pas disposer d'un droit de vote et doit être élu à l'unanimité des membres du conseil. Ainsi, le conseil d'administration est censé changer de nature et de mode de fonctionnement en devenant une instance de décision neutre chargé à la fois de sécuriser les investissements spécifiques en capital financier et en capital humain et chargé de sélectionner les couplages efficaces de capital financier et de capital humain.

\subsection{Qu'est-ce qu'une entreprise bien gouvernée ?}

A partir des mêmes questionnements sur la question de la propriété et du contrôle initiés par l'ouvrage fondateur de Berle et Means, la gouvernance de médiation apporte des réponses très différentes par rapport à celles apportées par la théorie de l'agence. Tout en intégrant la séparation de la propriété et du contrôle, la gouvernance de médiation offre une rupture axiomatique qui débouche sur une vision renouvelée de la gouvernance des entreprises. Ce changement d'axiomatique a trois conséquences importantes sur la manière de concevoir et pratiquer la gouvernance d'entreprise.

1- Le principal n'est pas l'actionnaire mais l'entreprise. Les actionnaires ne sont pas propriétaires de l'entreprise et par conséquent il n'est pas possible et juridiquement valide de leur reconnaitre une souveraineté. Comme le souligne Lan et Heracléous, cet argument aux fondements de la théorie de l'agence est basé sur une "mauvaise interprétation de la nature de la propriété des actions. Il ignore le principe légal de personnification de la société, de la responsabilité limité des actionnaires et de la responsabilité potentiellement illimitée des administrateurs" (Lan \& Heracleous, 2010, p. 301). Les actionnaires ne constituant pas la partie prenante souveraine, ils ne peuvent en aucun cas orienter et récupérer l'intégralité de la valeur économique dégagée. Ils ne peuvent pas être considérés comme le principal de la relation d'agence qui s'établit avec les dirigeants et les administrateurs puisque le principal est l'entreprise elle-même.

2- Les administrateurs ne sont plus les agents des actionnaires. Comme le souligne Blair \& Stout "les administrateurs ne sont pas, en droit, les agents de quiconque... Les administrateurs ne font pas obédience aux actionnaires ni à d'autres acteurs' ((Blair 
\& Stout, 2001, pp. 423-424). Après avoir contesté l'identité du principal, il est assez logique que la gouvernance de médiation offre une alternative importante sur le rôle et la contribution des administrateurs. Ces derniers sont des agents fiduciaires, des mandataires en charge de l'intérêt social et du développement de l'entreprise. Ils agissent au nom et dans l'intérêt de l'entreprise et pas en tant qu'agents des actionnaires.

\section{3- Le rôle du conseil d'administration n'est pas de contrôler et ni de sanctionner les}

dirigeants. La mission essentielle du conseil d'administration est de trouver des équilibres entre les différents apporteurs de capitaux spécifiques et pas de contrôler et aligner les intérêts des dirigeants avec ceux des actionnaires. Il doit s'assurer d'une implication maximale des différents apporteurs de ressources et compétences et répartir équitablement la valeur générée. Il doit trouver des équilibres dans les revendications et intérêts des investisseurs spécifiques, répartir équitablement le surplus via un contrôle des actifs de la société. Le conseil d'administration ne doit pas être réduit à un simple organe de contrôle à la solde des actionnaires mais comme une instance de pilotage d'un nœud d'investissements spécifiques (Blair \& Stout, 2001; Stout, 2007).

Nous résumons dans le tableau 1 ci-dessous les différences des deux cadres théoriques sur les points centraux que constituent la propriété et la nature de l'entreprise ainsi que le rôle du conseil d'administration. 


\begin{tabular}{|c|c|c|}
\hline & Théorie de l'agence & Gouvernance de médiation \\
\hline Propriété & $\begin{array}{c}\text { Les actionnaires sont propriétaires } \\
\text { de l'entreprise. Ils sont souverains } \\
\text { et possèdent un droit sur la } \\
\text { créance résiduelle. }\end{array}$ & $\begin{array}{c}\text { L'entreprise n'est pas un objet de } \\
\text { propriété. Elle n'appartient à } \\
\text { personne. }\end{array}$ \\
\hline $\begin{array}{c}\text { Nature de } \\
\text { l'entreprise }\end{array}$ & $\begin{array}{c}\text { L'entreprise n'existe pas. C'est une } \\
\text { fiction légale qui sert de support } \\
\text { juridique à un noud de relations } \\
\text { contractuelles. }\end{array}$ & $\begin{array}{c}\text { L'entreprise est un noud } \\
\text { d'investissements spécifiques qui } \\
\text { génère une quasi-rente } \\
\text { relationnelle. }\end{array}$ \\
\hline $\begin{array}{c}\text { Rôle du conseil } \\
\text { d'administration }\end{array}$ & $\begin{array}{c}\text { Les administrateurs sont les agents } \\
\text { des actionnaires et doivent } \\
\text { contrôler, orienter et sanctionner } \\
\text { l'action des dirigeants en faveur } \\
\text { des actionnaires }\end{array}$ & $\begin{array}{c}\text { Le conseil d'administration doit } \\
\text { s'assurer d'un investissement } \\
\text { maximal des apporteurs de } \\
\text { ressources spécifiques et veuillez à } \\
\text { une répartition équitable de la } \\
\text { valeur ajoutée }\end{array}$ \\
\hline
\end{tabular}

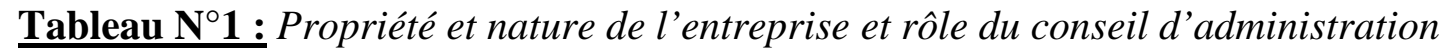

\section{Les développements récents et l'appropriation par les gestionnaires}

Le cadre théorique de la gouvernance de médiation renoue avec un questionnement initié par l'ouvrage de Berle et Means qui demeure d'actualité : quels intérêts une entreprise doit-elle servir? Quelle est sa nature ? Quel sont les rôles et mandats des administrateurs ? Le cadre théorique a connu un certains nombre de développement depuis les années 1990 qui sont emblématiques de son identité fondatrice au cœur de l'économie et du droit. Il fait l'objet d'une appropriation plus récente par les gestionnaires qui voient dans ce cadre théorique une opportunité pour répondre à la crise de légitimité de la gouvernance actionnariale et proposer une nouvelle vision de l'entreprise.

\subsection{Les développements en droit}

Comme nous l'avons noté plus haut, la gouvernance de médiation prend ces racines au carrefour du droit et de l'économie et l'article fondateur rédigé en 1999 par Blair et Stout est avant tout un article de droit. Une partie des auteurs attachés à la gouvernance de médiation a ainsi développé des travaux de nature juridique pour à la fois montrer les faiblesses de la théorie de l'agence mais également les possibilités de réformes du droit constitutionnel américain pour accompagner une évolution des pratiques de gouvernance. Ces travaux en droit sont en particulier menés par Blair et Stout qui rappellent inlassablement les erreurs 
commises par les défenseurs de la théorie de l'agence et la nécessité de corriger le droit des sociétés américains (Stout, 2012).

En France, les travaux de Jean-Philippe Robé rappellent avec constance que l'entreprise n'est pas un objet de propriété et qu'on ne peut en aucun cas affirmer que les actionnaires sont les propriétaires légitimes. Les travaux de Robé se positionnent plus particulièrement sur la question de la grande entreprise internationalisée qui pose des problèmes importants de régulation. Les pouvoirs acquis par les grandes entreprises nécessitent un important travail juridique de la part des Etats pour repenser leurs pratiques de gouvernance ainsi que leurs responsabilités sociales (Robé, 1997, 1999, 2001, 2009a, 2009b, 2010, 2011a, 2011b, 2014). En résonnance avec ces travaux, le collège des Bernardins a lancé en 2012 un programme de recherche sur la propriété, la création collective et le monde commun qui visait à développer des réflexions sur les formes de la propriété et les responsabilités sociales des entreprises. Ce programme a donné lieu à des publications au croisement du droit et du management qui invitent à repenser les mécanismes de création et de partage de la valeur dans la gouvernance des entreprises contemporaines (Barreto, 2012; Favereau \& Roger, 2015; Le Gall, 2011; Segrestin \& Hatchuel, 2012; Segrestin, Levillain, Vernac, \& Armand, 2015; Segrestin et al., 2014).

Ces travaux ont directement inspiré la loi PACTE portant sur l'entreprise nouvelle et les nouvelles gouvernances (Hollandts \& Valiorgue, 2018a, 2018b, 2018c). Cette proposition de loi fait le constat des dérives générées par la gouvernance actionnariale et la théorie de l'agence et propose un cadre législatif adapté au fonctionnement réel des entreprises et leurs pouvoirs d'influence sur la société civile. Les porteurs de cette loi rappellent en toute cohérence avec les travaux sur la gouvernance de médiation que les actionnaires ne pas propriétaire de l'entreprise mais d'actions et que ces derniers ne sont pas les seuls à être exposés à des risques. Fort de ce double constat, une des propositions phares de la loi consiste à transformer en profondeur la norme fondamentale de gestion de l'entreprise en prévoyant que «la société (qui supporte l'activité de l'entreprise) doit être gérée conforment à son objet social, en tenant compte des conséquences économiques, sociales et environnementales de son activité ». Le législateur français semble avoir pris conscience des impasses politiques et théoriques du modèle actionnarial et, à travers la loi PACTE, il a enclenché un travail de remise en cause du modèle actionnarial afin de renforcer les démarches socialement responsables et la prise en charge des externalités négatives (Daudigeos \& Valiorgue, 2010a; Hollandts \& Valiorgue, 2012; Valiorgue, 2016) 


\subsection{Les développements en économie politique}

Dans les années 2000, les travaux de Rajan et Zingales ont donné lieu à une expansion considérable de la gouvernance de médiation et une visibilité importante du cadre théorique en dehors des débats en droit. Les deux auteurs et en particulier Zingales ont mobilisé les développements de la gouvernance de médiation dans un double mouvement. Il s'agit dans les deux cas de montrer la fragilité conceptuelle et les effets pervers de la théorie de l'agence.

Les premiers travaux montrent comment les développements autour de la gouvernance de médiation peuvent conduire à une nouvelle théorie de l'entreprise qui intègre les mécanismes de création de valeur et la stabilisation d'une rentre relationnelle (Rajan \& Zingales, 1998, 2000, 2001; Zingales, 2000). Rajan et Zingales montrent que la conception de l'entreprise comme simple nœud de contrats ne permet pas de rendre compte de la complexité des mécanismes à l'œuvre et tend à faire de l'entreprise une simple variante du marché. Ils s'appuient alors sur les premières réflexions de M. Blair mais également sur la théorie des contrats incomplets pour montrer que l'entreprise est avant tout une collection d'actifs spécifiques dont il faut gouverner la cohérence et la pleine et entière implication. Ils en déduisent une nouvelle conception de l'autorité de gestion et un rôle nouveau pour le conseil d'administration qui doit se positionner en arbitre des conflits potentiels entre les apporteurs de ressources et capitaux spécifiques.

Cette nouvelle théorie de l'entreprise et les principes de gouvernance qui en découlent est ensuite utilisée par les auteurs pour démontrer les effets négatifs de la gouvernance actionnariale sur la performance de l'économie américaine. Les auteurs montrent que la gouvernance actionnariale conduit à une sur-captation de la valeur économique par les actionnaires et réduit la contribution des autres parties prenantes. Il y a pour les auteurs une forme de «hold-up actionnarial » à travers la gouvernance actionnariale qui décourage les autres investisseurs spécifiques car ces derniers ne reçoivent qu'une faible partie de la valeur qu'ils ont contribué à créer. En donnant trop d'importance et de poids aux actionnaires à travers la théorie de l'agence et l'institutionnalisation de la gouvernance actionnariale, Rajan et Zingales montrent que c'est le dynamisme même du capitalisme américain qui est remis en question (Rajan \& Zingales, 2003; Zingales, 2014). Il est clair pour les deux auteurs que cette prépondérance pour les actionnaires entraine une perte de dynamisme et une diminution de la capacité d'innovation des entreprises qui se traduit in fine par une perte de richesse collective à l'échelle du système économique. Il s'en suit alors une série de propositions de réformes 
pour rééquilibrer les choses en matière de gouvernance et mettre en œuvre les principes d'une gouvernance de médiation.

Plus récemment, L. Zingales a pris en 2015 la tête du Stigler Center et mène des travaux sur le «crony capitalism ». Le centre de recherche étudie les interactions entre l'économique et le politique afin de montrer comment la régulation et les équilibres concurrentiels sont subvertis par les intérêts particuliers au détriment du bien-être général. Zingales tire ici les conséquences d'une domination hégémonique de la théorie de l'agence depuis plus de 40 ans et s'attèle désormais à montrer comment les enjeux de régulation et en particulier les principes de gouvernance sont détournés au profit d'intérêt privés ${ }^{13}$.

\subsection{Une appropriation progressive et tardive par les gestionnaires}

A l'exception de quelques auteurs, les gestionnaires ont mis du temps à découvrir et s'intéresser à la gouvernance de médiation. Les premières publications datent pour l'essentiel des années 2010 et il est intéressant de voir qu'il s'agit plutôt de chercheurs européens qui mobilisent le cadre théorique. L'article de Lan et Heracleous publié en 2010 dans l'Academy of Management Review a permis de donner une visibilité importante à la gouvernance de médiation dans la communauté des chercheurs en management et ouvrir des débats sur les effets et conséquences de la théorie de l'agence (Heracleous \& Lan, 2012; Lan \& Heracleous, 2010). Assez rapidement, d'autres chercheurs se sont emparés du questionnement dans une perspective essentiellement critique afin de dénoncer les conséquences managériales, organisationnelles et sociétales de la théorie de l'agence (Baars \& Spicer, 2017). Cependant, les gestionnaires s'approprient non sans une certaine maladresse les questions autour de la propriété de l'entreprise et retombent assez vite dans les débats qui opposent théorie de l'agence et théorie des parties prenantes. A l'exception notable des recherches menées à l'école des Mines autour d'Hatchuel et Segrestin peu d'auteurs en gestion s'intéressent à la question centrale qui est cœur des questions de gouvernance depuis Berle et Means et réactiver par la gouvernance de médiation : les mécanismes de création et partage de la valeur (Segrestin \& Hatchuel, 2012; Segrestin et al., 2015). Contrairement à certains juristes et économistes, les gestionnaires semblent avoir du mal à tirer pleinement les conséquences d'un changement de paradigme en matière de gouvernance des entreprises pour orienter et structurer de nouvelles problématiques, objets et méthodes de recherche.

\footnotetext{
${ }^{13}$ https://research.chicagobooth.edu/stigler/
} 


\section{Conclusion}

Le siècle dernier a permis de faire émerger dans le débat public et académique la question de la gouvernance de l'entreprise et de son orientation. Mis au jour par Berle et Means, la gouvernance de l'entreprise s'est peu à peu articulée autour du seul référentiel théorique de la théorie de l'agence qui s'est imposé de manière hégémonique depuis la fin des années 1970. En dépit des nombreuses controverses et dommages collatéraux générés par ces principes de gouvernance, c'est à l'heure actuelle ce cadre théorique qui oriente les réflexions, alimentent la production de textes juridiques et la formation voire la vision des acteurs de la gouvernance. En dehors de controverses essentiellement académiques et théoriques, la gouvernance de médiation dont la validité juridique et le potentiel heuristique sont incontestablement supérieurs, n'est pas arrivée à orienter le débat public, la production de textes de lois et la recherche en management.

Plusieurs raisons peuvent expliquer ce manque d'audience de la gouvernance de médiation. Premièrement, on ne peut qu'être admiratif de l'extraordinaire simplicité et parcimonie offertes par la théorie de l'agence. Le cadre théorique développe une vision simple et cohérente de l'entreprise et des questions de gouvernance (Gomez, 1996). Par ailleurs, on peut noter que la théorie de l'agence s'arrime très bien aux valeurs essentielles des sociétés contemporaines (propriété privée, incitation, libéralisme, individualisation). De plus, les principaux auteurs relevant de ce courant ont pu bénéficier d'une exposition académique et médiatique importante en raison du fait qu'ils appartenaient à des institutions disposant d'une certaine visibilité et en bénéficiant de relais importants, dans le monde des affaires notamment. Le fait, que ces mêmes auteurs aient pu également former des générations entières de managers et de futurs dirigeants a également contribué à la diffusion progressive des thèses liées à la théorie de l'agence. Au final, on ne peut que constater la présence hégémonique de la théorie de l'agence tant dans les débats académiques que dans les pratiques. La théorie de l'agence a donc, sans contestation, fait preuve d'une grande performativité qui est sans équivalent dans les sciences sociales.

Il n'en demeure pas moins que les conséquences directes et indirectes de cette performativité sont nombreuses. En effet, comment ne pas voir l'augmentation considérable de la rémunération des dirigeants, au-delà de toute logique et rationalité, comme une dérive du cadre offert par la théorie de l'agence, mettant en cause les principes de solidarité et de justice sociale nécessaire à la société ? Comment ne pas déplorer l'évaluation permanente des 
entreprises, sommées de publier des résultats trimestriels, ne correspondant à aucune logique économique, industrielle ou financière? Comment enfin accepter qu'une entreprise puisse perdre la moitié de sa valeur en deux jours pour une simple alerte sur résultats, comme Toshiba fin 2016 ? Au cœur de ces interrogations, que certains analysent comme des dérives, il y a «l'idolâtrie» de la valeur actionnariale, qui apparait comme la seule boussole des actionnaires et des dirigeants et par ricochet des salariés et des autres parties prenantes.

Derrière les problématiques de gouvernance, les enjeux apparaissent comme cruciaux et n'ont pas fondamentalement changé depuis l'ouvrage de Berle et Means : qui contrôle les grandes entreprises ? Quels sont les intérêts servis ? Comment sont-elles contrôlées ? Comment les risques sont traités et appréhendés ? Quelles sont les moyens pour contester les décisions prises de la part de ceux qui les subissent ? La gouvernance de médiation que nous avons exposée dans le cadre de ce chapitre propose un cadre d'analyse plus équilibré et plus inclusif mais également plus conforme à ce qu'est une entreprise en droit. En cela, la gouvernance de médiation rompt avec «l'imposture » ou la méprise théorique entretenue par la théorie de l'agence. La question qui se pose par la suite est pourquoi la gouvernance de médiation n'a pas, en raison de son apport (et de son ethos), remplacé peu à peu la théorie de l'agence. Audelà du changement paradigmatique, la gouvernance de médiation souffre sans doute de son apparente complexité, qui la rend plus impropre à une diffusion rapide, à la fois dans la sphère académique et professionnelle. Par ailleurs, l'opérationnalité de la fonction du conseil d'administration semble pour l'heure compliquée à mettre en œuvre dans le cadre des conseils existants. Les prochains travaux devront ainsi montrer comment le cadre s'opérationnalise dans des contextes entrepreneuriaux où c'est avant tout l'intérêt de l'entreprise et des investisseurs spécifiques qui est privilégié. Les chercheurs en management stratégique ont à cet égard une responsabilité bien particulière. Leurs travaux doivent mieux montrer comment l'opérationnalisation d'une gouvernance de médiation permet de stabiliser des modèles économiques performants et inclusifs. Loin de se réduire à une simple délégation de pouvoir entre acteurs réputés propriétaires et dirigeants, l'articulation gouvernance - stratégie est au cœur du processus entrepreneurial et c'est de cette articulation que dépend la capacité pour les acteurs de bâtir et mettre en œuvre des stratégies durables (Acquier, Daudigeos, \& Valiorgue, 2011; Daudigeos \& Valiorgue, 2010b; Martinet \& Reynaud, 2015). 


\section{Bibliographie}

Acquier, A., Daudigeos, T., \& Valiorgue, B. (2011). Corporate social responsibility as an organizational and managerial challenge: the forgotten legacy of the Corporate Social Responsiveness movement. M@n@gement, 14(4), 222-250.

Aghion, P., \& Holden, R. (2011). Incomplete contracts and the theory of the firm: What have we learned over the past 25 years? The Journal of Economic Perspectives, 25(2), 181-197.

Alchian, A. A. (1961). Some economics of property: Rand Corporation Santa Monica, CA.

Aoki, M. (2000). Information, corporate governance and institutional diversity: Competitiveness in Japan, the USA, and the transitional economies: Oxford University Press, Inc.

Baars, G., \& Spicer, A. (2017). The Corporation: A Critical, Multi-Disciplinary Handbook: Cambridge University Press.

Barreto, T. (2012). L'entreprise, formes de la propriété et responsabilités sociales: Lethielleux.

Barzel, Y. (1997). Economic analysis of property rights: Cambridge university press.

Berle, A. A., \& Means, G. G. (1932). The modern corporation and private property: Transaction publishers.

Blair, M. M. (1996). Ownership and control: Rethinking corporate governance for the twenty-first century: Brookings Institution.

Blair, M. M., \& Stout, L. A. (1999). A team production theory of corporate law. Virginia Law Review, 247-328.

Blair, M. M., \& Stout, L. A. (2001). Director accountability and the mediating role of the corporate board. Wash. ULQ, 79, 403.

Charreaux, G. (1997). Le gouvernement des entreprises: Corporate Governance: théories et faits.

Charreaux, G., \& Desbrières, P. (1998). Gouvernance des entreprises: valeur partenariale contre valeur actionnariale. Finance Contrôle Stratégie, 1(2), 57-88.

Chassagnon, V., \& Hollandts, X. (2014). Who are the owners of the firm: shareholders, employees or no one? Journal of Institutional Economics, 10(01), 47-69.

Connelly, B. L., Hoskisson, R. E., Tihanyi, L., \& Certo, S. T. (2010). Ownership as a form of corporate governance. Journal of Management Studies, 47(8), 1561-1589.

Daily, C. M., Dalton, D. R., \& Cannella, A. A. (2003). Corporate governance: Decades of dialogue and data. Academy of management review, 28(3), 371-382.

Daudigeos, T., \& Valiorgue, B. (2010a). Les liens incertains entre RSE et création de valeur marchande. Revue française de gestion, 203(4), 25-39.

Daudigeos, T., \& Valiorgue, B. (2010b). Les limites du traitement marchand de la Responsabilité sociale de l'entreprise. Revue française de socio-économie, 6(2), 65-86.

Davis, G. F. (2009). Managed by the markets: How finance re-shaped America. Oxford: Oxford University Press.

Ellerman, D. P. (1990). Perestroika with worker ownership. Annals of Public and Cooperative Economics, 61(4), 519-535.

Fama, E. F., \& Jensen, M. C. (1983). Separation of ownership and control. The journal of law and Economics, 26(2), 301-325.

Favereau, O., \& Roger, B. (2015). Penser l'entreprise: nouvel horizon du politique: Éditions Parole et silence.

Foss, K., \& Foss, N. (2015). Coasian and modern property rights economics. Journal of Institutional Economics, 11(2), 391-411.

Frey, B. S., \& Osterloh, M. (2005). Yes, managers should be paid like bureaucrats. Journal of Management Inquiry, 14(1), 96-111.

Furubotn, E. G., \& Pejovich, S. (1972). Property rights and economic theory: a survey of recent literature. Journal of economic literature, 10(4), 1137-1162.

Gindis, D. (2007). Some building blocks for a theory of the firm as a real entity.

Gindis, D. (2016). Legal personhood and the firm: avoiding anthropomorphism and equivocation. Journal of Institutional Economics, 12(3), 499-513.

Gomez, P. (2003). Jalons pour une histoire des théories du gouvernement des entreprises. Finance Contrôle Stratégie, 6(4), 183-208. 
Gomez, P. Y. (1996). Le gouvernement de l'entreprise: modèles économiques de l'entreprise et pratiques de gestion. Paris: InterEditions.

Gomez, P. Y. (2001). La République des Actionnaires: le gouvernement des entreprises, entre démocratie et démagogie. Paris: Syros.

Hansmann, H., \& Kraakman, R. (2001). The end of history for corporate law. Geogetown Law Journal, 89, 439-468.

Heracleous, L., \& Lan, L. L. (2012). Agency theory, institutional sensitivity, and inductive reasoning: Towards a legal perspective. Journal of Management Studies, 49(1), 223-239.

Herrigel, G. (2007). Guest editor's introduction: A new wave in the history of corporate governance. Enterprise \& Society, 8(3), 475-488.

Hillman, A. J., \& Keim, G. D. (2001). Shareholder value, stakeholder management, and social issues: what's the bottom line? Strategic Management Journal, 125-139.

Hollandts, X., \& Valiorgue, B. (2012). La RSE comme processus entrepreneurial de conversion de valeurs sociales en valeur marchande: un examen empirique du lien entre engagements sociétaux et performance économique des entreprises françaises cotées entre 1999 et 2009. Revue de l'organisation responsable, 6(2), 20-37.

Hollandts, X., \& Valiorgue, B. (2018a). Loi PACTE: favoriser les entrepreneurs politiques, pour le meilleur et pour le pire. Retrieved from https://theconversation.com/loi-pacte-favoriser-lesentrepreneurs-politiques-pour-le-meilleur-et-pour-le-pire-92988

Hollandts, X., \& Valiorgue, B. (2018b). Pour un «comité des parties prenantes spécifiques» au sein des conseils d'administration. Retrieved from https://theconversation.com/reforme-delentreprise-pour-un-comite-des-parties-prenantes-specifiques-au-sein-des-conseilsdadministration-91582

Hollandts, X., \& Valiorgue, B. (2018c). Redéfinir l'entreprise et sa finalité: une révolution en marche ? Retrieved from https://theconversation.com/redefinir-lentreprise-et-sa-finalite-unerevolution-en-marche-89419

Jensen, M. C., \& Meckling, W. H. (1976). Theory of the firm: Managerial behavior, agency costs and ownership structure. Journal of financial economics, 3(4), 305-360.

L'Hélias, S. (1997). Le retour de l'actionnaire. Pratiques du corporate governance en France, aux États-Unis et en Grande-Bretagne, Paris, Gualino éditeur.

Lan, L. L., \& Heracleous, L. (2010). Rethinking agency theory: The view from law. Academy of management review, 35(2), 294-314.

Lazonick, W., \& O'sullivan, M. (2000). Maximizing shareholder value: a new ideology for corporate governance. Economy and Society, 29(1), 13-35.

Le Gall, J.-M. (2011). L'entreprise irréprochable: réciprocité, responsabilité, démocratie: Desclée de Brouwer.

MacKenzie, D., Muniesa, F., \& Siu, L. (2007). Do economist make markets? On the performativity of economics: Princeton University Press, Princeton.

Martinet, A.-C., \& Reynaud, E. (2015). Shareholders, stakeholders et stratégie. Revue française de gestion(8), 297-317.

Osterloh, M., \& Frey, B. S. (2006). Shareholders should welcome knowledge workers as directors. Journal of Management and Governance, 10(3), 325-345.

Pérez, R. (2010). La gouvernance de l'entreprise. Paris: La découverte.

Rajan, R., \& Zingales, L. (2003). Saving capitalism from the capitalists: Princeton University Press

Rajan, R. G., \& Zingales, L. (1998). Power in a Theory of the Firm. The Quarterly Journal of Economics, 113(2), 387-432.

Rajan, R. G., \& Zingales, L. (2000). The governance of the new enterprise. Retrieved from

Rajan, R. G., \& Zingales, L. (2001). The firm as a dedicated hierarchy: A theory of the origins and growth of firms. The Quarterly Journal of Economics, 116(3), 805-851.

Ripert, G. (1946). Aspects juridiques du capitalisme modern: Librarie générale de droit et de jurisprudence.

Robé, J.-P. (1997). Multinational enterprises: the constitution of a pluralistic legal order. Global Law Without A State. Aldershot: Dartmouth Gower, 45-77.

Robé, J.-P. (1999). L'entreprise et le droit: Presses universitaires de France.

Robé, J.-P. (2001). L'entreprise oubliée par le droit. Journal de l'Ecole de Paris, 32, 29-37. 
Robé, J.-P. (2009a). À qui appartiennent les entreprises? Le débat(3), 32-36.

Robé, J.-P. (2009b). Responsabilité limitée des actionnaires et responsabilité sociale de l'entreprise. Entreprises et histoire(4), 165-183.

Robé, J.-P. (2010). Les États, les entreprises et le droit. Le débat(4), 74-87.

Robé, J.-P. (2011a). L'entreprise et la constitutionnalisation du système-monde de pouvoir. Collège des Bernardins-département Economie, Hommes, Société.

Robé, J.-P. (2011b). The legal structure of the firm. Accounting, Economics, and Law, 1(1).

Robé, J.-P. (2014). Comment s' assurer que les entreprises respectent l'intérêt général. L'Économie politique(4), 22-35.

Segrestin, B. (2011). Une nouvelle «Modern Corporation»: relecture gestionnaire de l'ouvrage de Berle \& Means. Paper presented at the Annales des Mines-Gérer et comprendre.

Segrestin, B., \& Hatchuel, A. (2009). L'entreprise, une invention moderne en attente de droit? Entreprises et histoire(4), 218-233.

Segrestin, B., \& Hatchuel, A. (2012). Refonder l'entreprise. Paris: Seuil.

Segrestin, B., Levillain, K., Vernac, S., \& Armand, H. (2015). La Société à Objet Social Étendu : proposition pour un nouveau statut de l'entreprise. Paris: Presse des Mines.

Segrestin, B., Roger, B., \& Vernac, S. (2014). L'Entreprise: Point aveugle du savoir. Paris: Sciences Humaines.

Silverman, B. S., \& Ingram, P. (2017). Asset ownership and incentives in early shareholder capitalism: Liverpool shipping in the eighteenth century. Strategic Management Journal, 38(4), 854-875.

Stout, L. A. (2007). The mythical benefits of shareholder control. Virginia Law Review, 789-809.

Stout, L. A. (2008). Why we should stop teaching Dodge v. Ford. Va. L. \& Bus. Rev., 3, 163.

Stout, L. A. (2012). The shareholder value myth: How putting shareholders first harms investors, corporations, and the public: Berrett-Koehler Publishers.

Valiorgue, B. (2016). Actions collectives et traitement des externalités négatives: Université d'Auvergne.

Veldman, J., \& Willmott, H. (2017). Social ontology and the modern corporation. Cambridge Journal of Economics, 41(5), 1489-1504.

Zingales, L. (2000). In search of new foundations. The Journal of Finance, 55(4), 1623-1653.

Zingales, L. (2014). A capitalism for the people: Recapturing the lost genius of American prosperity: Basic books. 